\title{
Gravity without Newton's Gravitational Constant and No Knowledge of Mass Size
}

\author{
Espen Gaarder Haug \\ Norwegian University of Life Sciences - Chr. Magnus Falsens Road, 1430 Ås, Norway \\ (Dated: August 11, 2018)
}

\begin{abstract}
In this paper we show that the Schwarzschild radius can be extracted easily from any gravitationally-linked phenomena without having knowledge of the Newton gravitational constant or the mass size of the gravitational object. Further, the Schwarzschild radius can be used to predict any gravity phenomena accurately, again without knowledge of the Newton gravitational constant and also without knowledge of the size of the mass, although this may seem surprising at first.

Hidden within the Schwarzschild radius are the mass of the gravitational object, the Planck mass (their relative mass), and the Planck length. We do not claim to have all the answers, but this seems to indicate that gravity is quantized, even at a cosmological scale, and this quantization is directly linked to the Planck units. This also supports our view that the Newton gravitational constant is a universal composite constant of the form $G=\frac{l_{p}^{2} c^{3}}{\hbar}$, rather than relying on the Planck units as a function of $G$. This does not mean that Newton's gravitational constant is not a universal constant, but that it is instead a composite universal constant that depends on the Planck length, the speed of light, and the Planck constant. Further, $\frac{G \times 1 \text { weight unit }}{c^{2}}=\frac{G}{c^{2}}$ is the Schwarzschild radius off one weight unit. So $G$ is only needed when we want to use gravity to find the weight of an object, such as weighing the Earth.

This is, to our knowledge, the first paper that shows how a long series of major gravity predictions and measurements can be completed without any knowledge of the mass size of the object, or Newton's gravitational constant. As a minimum we think it provides an interesting new angle for evaluating existing gravity theories, and it may even give us a small hint on how to combine quantum gravity with Newton and Einstein gravity.

Key words: Schwarzschild radius, Weight, Planck mass, Planck length, measurement, gravitational constant, Heisenberg.
\end{abstract}

\section{INTRODUCTION}

We will start this paper on what some may think is a slightly unscientific tone, but we believe it is well-suited for a short introduction to what we will show later on. In subsequent sections we will expand on the key points with more rigorous derivations and scientific principles.

Thus, let us assume that an alien came to Earth and gave you the following formula

$$
r_{e}=\frac{g R^{2}}{c^{2}}
$$

The alien explained that $g$ is the gravitational acceleration, $R$ is the radius from the center of the planet (gravitational mass) to the surface, and $c$ is the well-known speed of light. The gravitational acceleration is easy to measure without any knowledge of gravity (for example with two time-gates and a object in free fall); it is about $9.8 \mathrm{~m} / \mathrm{s}^{2}$. The radius of the Earth is not that easy to measure, but we know it is about 6,371,000 meters. As for the speed of light, we can measure it with a low cost kit and or just take the standard accepted speed, which is defined as exactly $299,792,458$ meters per second. The main point is that one needs no knowledge of gravity to measure each of these input factors.

Next we plug these values into the formula above and get

$$
r_{e}=\frac{9.8 \times 6371000^{2}}{299792458^{2}} \approx 0.00442588 \text { meter }
$$

Some will recognize that this is very similar to half of the value of the Schwarzschild radius of the Earth; this is not a coincidence, as that is exactly what it is, something we will return to soon. Next, we can use this value of $r_{e}$ and plug it into any of the formulas below to calculate almost any major gravity predictions. We can predict the orbital velocity of a satellite, or the moon, for example, by the formula

$$
v_{o}=c \sqrt{\frac{r_{e}}{R_{o}}}
$$

where $R_{o}$ is the radius from the center of the Earth to the object for which we want to predict orbital velocity. Further, the time dilation between two clocks at different altitudes around a planet is given by

$$
\frac{T_{h}}{T_{L}}=\frac{\sqrt{1-2 \frac{r_{e}}{R_{L}}}}{\sqrt{1-2 \frac{r_{e}}{R_{h}}}}
$$

where $R_{h}$ is the radius further from the center of the earth than $R_{L}$. We can test this by placing one atomic clock at sea level and one at the top of a 2,000-meter mountain top. We naturally need to synchronize the 
clocks before performing this task. The clocks will be consistent with our gravity prediction. Again, all we need is $r_{e}$, which we can easily extract from the gravitational acceleration on the surface of the Earth, as already shown.

Next we can predict the red-shift; it is given by using the following formula

$$
\lim _{R \rightarrow+\infty} z(R)=\frac{1}{\sqrt{1-\frac{2 r_{e}}{R}}}-1
$$

If both the emitter and receiver are inside the gravitational field and we focus on frequency rather than change in wavelength, we have the well-known formula

$$
f_{h}=f_{L} \frac{\sqrt{1-\frac{r_{e}}{R_{L}}}}{\sqrt{1-\frac{r_{e}}{R_{h}}}}
$$

So based on this we can accurately predict the results of experimental set ups equal to the Pound and Rebka experiment [1]. This is just one example of how we can perform a series of gravitational predictions that can be confirmed by experiment without any knowledge of Newton's gravitational constant or the mass size of any object. What we have relied on instead is $r_{e}$, which can simply be obtained from the gravitational acceleration at the surface of the Earth, the speed of light, and the radius of the Earth.

\section{THE SCHWARZSCHILD RADIUS IN A NEW PERSPECTIVE}

The Schwarzschild radius comes from the Schwarzschild metric [2, 3] solution to the Einstein field equation [4] and is given by

$$
r_{s}=\frac{2 G M}{c^{2}}
$$

where $G$ is the Newton's gravitational constant, $M$ is the mass of the object, and $c$ is the well-known speed of light. In other words, we need to know the mass of the object of interest and the Newton gravitational constant in order to find its Schwarzschild radius. The escape velocity [5] from a mass $M$ at the radius $R$ from the center of the mass is given by

$$
v_{e}=\sqrt{\frac{2 G M}{R}}
$$

When we replace the radius in the escape velocity with the Schwarzschild radius $r=r_{s}=\frac{2 G M}{c^{2}}$ we get

$$
v_{e}=\sqrt{\frac{2 G M}{\frac{2 G M}{c^{2}}}}=c
$$

So, if an object with mass $M$ is packed inside the Schwarzschild radius, then we have a mass where even light cannot escape from inside the radius. This phenomenon is often known as a black hole, and the Schwarzschild radius is linked to black holes in this way.

Any object we have observed directly in the sky or on Earth has mass where the radius is extending outside the Schwarzschild radius. In other words, no mass has directly been detected that has all of its mass inside the Schwarzschild radius (even though recent gravitational wave detections may have detected collisions of black holes, but we not will discuss that further in this paper).

What is important here is that neither the Newton gravitational constant, nor the mass of the cosmological or smaller-sized objects, needs to be known to find the Schwarzschild radius. The Schwarzschild radius can be found directly as described in the section above from the gravitational acceleration of the Earth, or directly from the measured orbital velocity of a satellite such as the moon simply by using the formula

$$
r_{s}=2 r_{e}=2 \frac{v_{o}^{2} R}{c^{2}}
$$

where $R$ is now the radius from the center of the Earth to the orbital object of interest. Further, $v_{o}$ is the "easily" observed orbital velocity of the moon, for example. Alternatively, we could use two atomic clocks, measure time dilation between them, and then plug the values into this formula to find the Schwarzschild radius

$$
r_{e}=R \sqrt{1-\frac{T_{0}^{2}}{T_{f}^{2}}}
$$

where $T_{f}$ is a far away clock from the gravity field and $T_{0}$ is a clock placed at radius $R$ relative to the gravitational object. In most cases, we do not actually have access to a far-away clock $T_{f}$ from Earth, but we can easily have two clocks on Earth at altitude $R_{L}$ and $R_{h}$, and from this we can easily calculate find $r_{e}$ by solving the following equation with respect to $r_{e}$

$$
\frac{T_{h}}{T_{L}}=\frac{\sqrt{1-2 \frac{r_{e}}{R_{L}}}}{\sqrt{1-2 \frac{r_{e}}{R_{h}}}}
$$

this gives

$$
r_{e}=\frac{R_{h} R_{L}\left(T_{h}^{2}-T_{L}^{2}\right)}{2\left(R_{L} T_{h}^{2}-R_{h} T_{L}^{2}\right)}
$$


In 2016, Haug [6] suggested that the gravitational constant is likely a universal composite constant of the form

$$
G=\frac{l_{p}^{2} c^{3}}{\hbar} \approx 6.67 \times 10^{-11} \mathrm{~m}^{3} \cdot \mathrm{kg}^{-1} \cdot \mathrm{s}^{-2}
$$

Which is basically identical to a similar composite constant suggested by McCulloch [8]. This leads to an evaluation of the Schwarzschild radius at a deeper level by

$$
r_{s}=\frac{G M}{c^{2}}=\frac{\frac{l_{p}^{2} c^{3}}{\hbar} M}{c^{2}}=2 N l_{p}
$$

where $N$ is the number of Planck masses in the mass $M$. This is not new in itself, but the idea that we can find the Schwarzschild radius with no knowledge off $G$, or even the mass and use this to predict "all" known gravity phenomena is new, to the best of our knowledge. What seems important for gravity phenomena is the measurement of $N$ and $l_{p}$, and in this case, we do not need to know $N$ separately (the number of Planck masses), or $l_{p}$ separately, but the combination of the two $N l_{p}$ will be sufficient.

\section{G IS RELATED TO A STANDARDIZED SCHWARZSCHILD RADIUS CALIBRATED TO WEIGHT}

We have shown in the section above that the Schwarzschild radius can be easily found without any knowledge of the mass size or Newton's gravitational constant. We have also claimed that the Newton's gravitational constant is a composite constant of the form $G=\frac{l_{p}^{2} c^{3}}{\hbar}$. Actually, in order to use the Schwarzschild radius to find the mass of the object in terms of weight, we need to standardize it in relation to our weight definition. If we use $k g$ to describe mass, we need the Schwarzschild radius for one $\mathrm{kg}$ and this is simply $\frac{2 G}{c^{2}}$. The gravitational constant divided by $c^{2}$ can be seen as a standardized unit of half the Schwarzschild radius in relation to weight

$$
r_{e, 1}=\frac{G M}{c^{2}}=\frac{G \times 1}{c^{2}}=\frac{G}{c^{2}}=\frac{\frac{l_{p}^{2} c^{3}}{\hbar}}{c^{2}}=\frac{1}{m_{p}} l_{p}=N_{1} l_{p}
$$

where $N_{1}$ is the number of Planck masses in one kg. This is the Schwarzschild radius of one weight unit of whatever weight unit we have decided to use. Since Newton's gravitational constant is a function of also the reduced Planck constant, it automatically adjusts if we use another weight measure; if we use pounds, then the reduced Planck constant will be in pounds and $G$ will be in pounds $\left(m^{3} \cdot\right.$ Pounds $\left.s^{-1} \cdot s^{-2}\right)$. Further, when we want to extract the wegith of an object from gravity observations we will need $G$, otherwise we never need it.
This means in cases of gravity predictions and observations where we are not always interested in finding the weight of the object, such as the weight of the Earth, can be done completely without Newton's gravitational constant. The gravitational constant was first indirectly found by Cavendish in 1798 [7] to measure the weight of the Earth, and that is indeed when one needs to find it, as one then needs to calibrate the model with respect to the weight measure units one is using. Today the $\mathrm{kg}$ is redefined in terms of the Planck constant, so it should be not shocking that Newton's composite constant in this view must contain the reduced Planck constant.

Any Schwarzschild radius contains the relative mass of the gravity object. That is the size relative to that of the Planck mass (multiplied by the Planck length). So, all gravity is clearly related to mass. However, it is only when we also want to know the weight of a given object that need to know the Newton gravitational constant ${ }^{1}$.

The relative weight, and thereby also the relative weight of any two cosmological objects, is simply their Schwarzschild radius divided by each other.

Only if we want to extract the weight of an object from gravity do we need to know the Schwarzschild radius per weight unit chosen. In other words, that is when big $G$ is needed. Some will possibly claim that the Schwarzschild radius naturally was discovered after Newton discovered the gravity constant, so the Schwarzschild radius cannot be the central entity for gravity. We will claim that Newton's formula, even if it is correct and wonderful (at least as a first approximation), has not been fully understood. It has always been a mystery exactly what the Newton gravitational constant is.

Now, by showing all that gravity phenomena (in cases where we do not need to find the weight of the object) can be found by using the Schwarzschild radius directly from any gravity observation phenomena, and then this again can be used to predict any other gravity phenomena (except from finding the weight of the object, where we need the Schwarzschild radius per weight unit which is related to $G$.).

\section{ARGUMENTS IN FAVOR OF NEWTON'S GRAVITATIONAL CONSTANT BEING A COMPOSITE CONSTANT}

There are several observations that clearly support the idea that the Newton gravitational constant is a composite constant; here is a selection of them:

1. If we "never" need the Newton gravitational constant for any gravitation observation, not even in

1 That we can also obtain by finding the Planck mass weight first in a Cavendish apparatus, or alternatively the $G$ from the Cavendish apparatus

2 Needs further investigation and confirmation; see [11] for more details. 
TABLE I. The table shows that the most common gravitational measurements and predictions can be done without any knowledge of Newton's gravitational constant. Is it not time to ask if the Newton gravitational constant is a composite constant? Only when we want to separate out the Planck units or the gravitational constant do we need to know the mass size of the gravitational object.

\begin{tabular}{|c|c|c|c|c|}
\hline What to measure/predict & Formula & How & Is it easy to do & $\begin{array}{l}\text { Knowledge } \\
\text { of mass size }\end{array}$ \\
\hline Half Schwarzschild radius & $r_{e}=\frac{v_{o}^{2} R_{o}}{c^{2}}$ & From orbital velocity & Yes & No \\
\hline Half Schwarzschild radius & $r_{e}=R \sqrt{1-\frac{T_{0}^{2}}{T_{f}^{2}}}$ & $\begin{array}{c}\text { From time dilation } \\
\text { needs high precision clocks }\end{array}$ & $\begin{array}{c}\text { Difficult } \\
\text { need far away clock }\end{array}$ & No \\
\hline Half Schwarzschild radius & $r_{e}=\frac{R_{h} R_{L}\left(T_{h}^{2}-T_{L}^{2}\right)}{2\left(R_{L} T_{h}^{2}-R_{L} T_{L}^{2}\right)}$ & $\begin{array}{c}\text { From time dilation } \\
\text { needs high precision clocks }\end{array}$ & Yes & No \\
\hline Half Schwarzschild radius & $r_{e}=\frac{R_{h} R_{L}\left(f_{h}^{2}-f_{L}^{2}\right)}{2\left(R_{L} f_{h}^{2}-R_{L} f_{L}^{2}\right)}$ & From red-shift & Yes & No \\
\hline Half Schwarzschild radius & $r_{e}=\frac{\delta R}{4}$ & From light-bending & $\begin{array}{c}\text { less so } \\
\text { "need" eclipse }\end{array}$ & No \\
\hline Gravitational acceleration field & $g=\frac{r_{e}}{R^{2}} c^{2}$ & Find $r_{e}$ first & Yes & No \\
\hline Orbital velocity & $v_{o}=c \sqrt{\frac{r_{e}}{R}}$ & Find $r_{e}$ first & Yes & No \\
\hline Gravitational red-shift & $\lim _{R \rightarrow+\infty} z(R)=\frac{r_{e}}{R}$ & Find $r_{e}$ first & Yes & No \\
\hline Bekenstein-Hawking luminosity & $P=\frac{1}{15360 \pi} \frac{\hbar c^{2}}{r_{e}^{2}}$ & Find $r_{e}$ first & Yes & No \\
\hline $\begin{array}{c}\text { Schwarzschild radius }^{\mathrm{a}} \\
\text { for the Cavendish sphere }\end{array}$ & $r_{s}=\frac{4 L \pi^{2} R^{2} \theta}{c^{2} T^{2}}$ & Cavendish apparatus & Yes & No \\
\hline Planck mass & $m_{p}=\sqrt{\frac{\hbar c M T^{2}}{L 2 \pi^{2} r^{2} \theta}}$ & Cavendish apparatus & Yes & Yes $^{\mathrm{b}}$ \\
\hline Planck length & $l_{p}=\sqrt{\frac{\hbar L 2 \pi^{2} R^{2} \theta}{M T^{2} c^{3}}}$ & Cavendish apparatus & Yes & $\mathrm{Yes}^{\mathrm{c}}$ \\
\hline Planck time & $t_{p}=\sqrt{\frac{\hbar L 2 \pi^{2} R^{2} \theta}{M T^{2} c^{5}}}$ & Cavendish apparatus & Yes & Yes $^{\mathrm{d}}$ \\
\hline Gravitational constant & $\begin{array}{c}G=\frac{L 2 \pi^{2} r^{2} \theta}{M T^{2}} \\
G=\frac{\hbar c}{m_{p}^{2}}=\frac{l_{p}^{2} c^{3}}{\hbar} \approx 6.67 \times 10^{-11}\end{array}$ & Cavendish apparatus & Yes & $\mathrm{Yes}^{\mathrm{e}}$ \\
\hline
\end{tabular}

${ }^{a}$ Be aware that this is not the Schwarzschild radius of the Earth, as can be found by using the formulas further up in the table. Instead, this is the Schwarzschild radius of the large lead balls in the Cavendish apparatus. In this formula, no mass is needed, in contrast to a situation where we need to find Newton's gravitational constant.

b Need to know the mass of the large lead ball in the Cavendish apparatus, but this can be done by simply weighing it.

c Same as footnote above.

d Same as footnote above.

e Same as footnote above. 
calibrating a model, does this imply that it is not central for gravity either? See Table 1 for a long series of gravity calculations and observations that all can be done without any knowledge of the Newton gravitational constant, or the size of the mass in question. Further, if we want to separate out the Planck units, we need to know the size of the mass of the gravity object, otherwise that is not necessary.

2. When we understand that the Newton gravitational constant is related to a standardized Schwarzschild radius with respect to our chosen weight measure, then things become more clear. The Newton gravitational constant is only needed when we want to use gravity to find the weight of an object, for example the weight of the Earth or the Sun. However, we could then have found the Planck mass in a Cavendish apparatus instead (and just as easily) without knowledge of big $G$.

3. The output units of Newton's gravitational constant are given by: $\mathrm{m}^{3} \cdot \mathrm{kg}^{-1} \cdot \mathrm{s}^{-2}$. It would seem very strange if something fundamental existed at the deepest level that is meters cubed, divided by $\mathrm{kg}$ and seconds squared. It cannot be excluded, but one should first undertake a serious attempt to find a simpler explanation. We will claim this strongly indicates that the Newton gravitational constant must be a composite universal constant consisting of more fundamental constants.

4. By re-formulating $G$ as a composite of the form $G=$ $\frac{l_{p}^{2} c^{3}}{\hbar}$, a long series of the Planck units are dramatically simplified and become more logical. For example, the Planck time is described as $t_{p}=\sqrt{\frac{G \hbar}{c^{5}}}$, but such formulas give minimal intuition. We may ask, what is the logical meaning of $c^{5}$ and what is the deeper logic behind the gravitational constant? When replacing $G$ with its composite form, we simply show the Planck time as $t_{p}=\frac{l_{p}}{c}$, so the time it takes for light to travel the Planck length - this is naturally known.

5. The Planck mass and the Planck length can be measured totally independent of any knowledge of the Newton gravitational constant, as recently shown by Haug $[9,10]$. This means the elements of a Newtonian composite gravitational constant all are known. It seems more logical that at a fundamental level there exists a unique and likely the shortest possible length with any real meaning, namely the Planck length, as well as the speed of light. In addition, we have the Planck constant that is more complex, but in all observable gravity phenomena the gravitational constant even cancels out, and we are left with the Schwarzschild radius (or half of this in many cases) as the essential thing we need to know and can measure easily. Again, this consists of the number of Planck masses times the Planck length in the gravity object of interest.

6. We can possibly derive a gravitational theory from scratch based on the Heisenberg uncertainty principle, that combined with the analysis given here, generates a long series of gravity equations that give correct predictions without any knowledge of the Newton gravitational constant. This is the topic for section $\mathrm{V}$.

\section{MCCULLOCH-HEISENBERG NEWTON EQUIVALENT GRAVITY}

We will also mention a recently-published way of deriving Newtonian equivalent gravity that is potentially linked to the above analysis. The analysis above is in no way dependent on this theory, but is possibly compatible with it. In 2014, McCulloch [8] derived Newton's gravitational force from Heisenberg's uncertainty principle. Although the method can be criticized, it provides an interesting perspective on the themes of this paper. Here we will give a short overview of his derivation and point out several valid questions that we will answer, at least in part.

Heisenberg's uncertainty principle [12] is given by

$$
\Delta p \Delta x \geq \hbar
$$

McCulloch goes on to say "Now $E=p c$ so" :

$$
\Delta E \Delta x \geq \hbar c
$$

This assumption only holds for the Planck momentum $E=p c=m_{p} c c$, in our opinion. Further, from equation 18, McCulloch suggests that

$$
F=\frac{1}{(\Delta x)^{2}} \sum_{i}^{n} \sum_{j}^{N}(\hbar c)_{i, j}
$$

where $\sum_{i}^{n}$ is the number of Planck masses in a smaller mass $m$ we are working with, and $\sum_{i}^{N}$ corresponds to the the number of Planck masses in the larger mass we are working with. From this, McCulloch gets the equation

$$
F=\frac{\hbar c}{m_{p}^{2}} \frac{m M}{(\Delta x)^{2}}
$$

Further, McCulloch replaces $\Delta x$ with the radius and points out that

$$
G=\frac{\hbar c}{m_{p}^{2}} \approx 6.67384 \times 10^{-11} \mathrm{~m}^{3} \cdot \mathrm{kg}^{-1} \cdot \mathrm{s}^{-2}
$$


This is equivalent to the empirically observed Newton gravitational constant. It should be observed that there are still large measurement errors in the gravitational constant; see [13-17].

This means his derivation is equivalent to the Newtonian gravity formula

$$
F=G \frac{m M}{r^{2}}
$$

Although the idea is promising, several aspects of this derivation and gravity concept should be questioned.

First of all, in a follow up paper McCulloch states, [18].

In the above gravitational derivation, the correct value for the gravitational constant $G$ can only be obtained when it is assumed that the gravitational interaction occurs between whole multiples of the Planck mass, but this last part of the derivation involves some circular reasoning since the Planck mass is defined using the value for $G$.

The Planck mass was first derived by Max Planck in $1899[19,20]$, who assumed that there were three fundamental universal constants, namely the speed of light, the Newton gravitational constant, and the reduced Planck constant. The Planck mass was given as $m_{p}=\sqrt{\frac{\hbar c}{G}}$. To find the Planck mass, we need to know $G$, as pointed out by McCulloch and the McCulloch derivation appears to rest on Newtonian theory.

However, as has recently been shown by Haug, this is not necessarily the case, as we can measure the mass $m_{p}$ directly using a Cavendish apparatus without any prior knowledge of Newton gravity theory or the Newtonian gravitational constant [10]. In that case, we need to know the Planck constant in addition to the speed of light. The Planck constant can be measured independent of any knowledge of the Newton gravitational constant, using the Kibble balance, for example; see [21-23]. The main point is that we do not need to know the Newton gravitational constant to find the Planck mass.

Secondly, how can a principle derived by Heisenberg to understand the uncertainty in quantum world be relevant to this area of physics? Haug has recently re-derived the Heisenberg uncertainty principle with a specific focus on the Planck scale [25]. He has shown that the uncertainty principle likely collapses at the Planck scale and should be replaced with a certainty principle in the special case of the Planck mass:

$$
m_{p} c l_{p}=\hbar
$$

that is, when we are at the Planck scale for a particle with position $l_{p}$. We may also ask, How can the Planck mass be relevant when we are working with cosmological objects? In other working papers, we have presented a model of how the Planck mass particle could be the building block of all other particles. This may sound absurd at first, as the Planck mass is so much larger than any known particle. However, recent research has indicated that mass at a deeper level can be seen as a Compton clock [26, 27]. This suggests that the Planck mass is related to the Planck time [28], and instead of looking for a very large mass (compared to any observed particle), we should be looking for a very small mass, approximately $1.17 \times 10^{-51} \mathrm{~kg}$.

One could also question why McCulloch has not used the Kennard [24] version of the Heisenberg principle, as it is commonly known today that the correct version of the uncertainty principle is $\Delta p \Delta x \geq \frac{\hbar}{2}$, rather than $\Delta p \Delta x \geq$ $\hbar$. Here, we have to understand again that the Planck mass is very unique. The Planck mass is the only mass where the momentum always is related to $m c$, rather than to a velocity $v$ that varies between 0 and up to, but less than, c. This is discussed in more detail by Haug [25]. Just as the bending of light needs a factor of 2 relative to Newton to fit experiments (and relative to GR), could it be that the Planck mass particle, which is linked to $c$ rather than $v$, is also linked to $\hbar$ rather than $\frac{\hbar}{2}$ ?. We do not claim to have all the answers on this topic, but think one should keep an open mind that around the Planck mass, in particular, there could be aspects that are not fully understood in relation to the Heisenberg uncertainty principle.

Finally, we may ask, "Is the work standing on solid theoretical ground when McCulloch transforms the mass momentum relation into an energy position relation?". Due to the Pauli objection [29], the energy time version of the uncertainty relation is not considered valid by many physicists, because according to Pauli one cannot find a time operator that is both Hermitian and selfadjoint. McCulloch does not need a time operator, as he does not use time, but instead uses position in relation to energy. However, due to the fact that energies have been proven to come in quanta, and there is typically assumed continuous position when deriving the Heisenberg principle, then the position energy operator will likely also not be Hermitian and self-adjoint with respect to energy. In other words, the McCulloch derivation could run into the Pauli objection. More likely we think that the energy position and the energy time version of the uncertainty principle first introduced by Heisenberg are actually valid. Further, several researchers have recently suggested ways to get around the Pauli objection; see [31-33].

\section{CONCLUSION}

We have shown how a long series of gravity predictions and measurements are totally independent of knowledge of the Newton gravitational constant, or the size of the mass in question. One important component is (half) of the Schwarzschild radius, which at a deeper level is 
the number of Planck masses in the gravitational object multiplied by the Planck length. However, for most gravitational observations and predictions we do not need to break down the Schwarzschild radius into these fundamental components.

We also show, that contrary to possibly beliefs, we do not need any knowledge of the mass of the gravity object or the Newton gravitational constant to find the Schwarzschild radius of a cosmological object, or even a small clump of matter on Earth. This strongly supports our recent view that the Newton gravitational constant is a composite constant of the form $G=\frac{l_{p}^{2} c^{3}}{\hbar}$. In understanding this, we may be a bit closer to understanding the link between the quantum world and the macroscopic world in terms of gravity. We have not shown any new predictions in gravity, but we think our fresh angle on existing theory is interesting and also relevant from a practical point of view.

Newton's gravitational constant divided by $c^{2}$ is the Schwarzschild radius of one standardized mass unit in terms of weight, for example, one kg. The Newton gravitational constant is only needed when we are using grav- ity observations to find the mass of an object in terms of a standardized weight measure. However, the same can be done by finding the Planck mass weight using a Cavendish apparatus instead, without any knowledge of Newton's gravitational constant.

If our theory is correct, then any student or researcher can now, based on only measuring the gravitational acceleration on Earth, perform a long series of accurate gravity predictions without any knowledge of $G$ or the mass size of the object. Well we should be more specific here, the relative mass size of an object, that is relative to the Planck mass is indeed hidden in the Schwarzschild radius, the mass ratio times the Planck length is independent on weight.

\section{ACKNOWLEDGMENTS}

Thanks to Victoria Terces for helping me edit this manuscript and thanks to Eric Thompson for useful comments.
[1] R. V. Pound and G. A. Rebka Jr. Gravitational redshift in nuclear resonance. Physical Review Letters, 3(9): 439-441, 1959.

[2] K. Schwarzschild. Über das gravitationsfeld eines massenpunktes nach der Einsteinschen theorie. Sitzungsberichte der Deutschen Akademie der Wissenschaften zu Berlin, Klasse fur Mathematik, Physik, und Technik, page 189, 1916.

[3] K. Schwarzschild. Über das gravitationsfeld einer kugel aus inkompressibler flussigkeit nach der Einsteinschen theorie. Sitzungsberichte der Deutschen Akademie der Wissenschaften zu Berlin, Klasse fur Mathematik, Physik, und Technik, page 424, 1916.

[4] A. Einstein. Näherungsweise integration der feldgleichungen der gravitation. Sitzungsberichte der Königlich Preussischen Akademie der Wissenschaften Berlin, 1916.

[5] A. T. Augousti and A. Radosz. An observation on the congruence of the escape velocity in classical mechanics and general relativity in a Schwarzschild metric. European Journal of Physics, 376:331-335, 2006.

[6] E. G. Haug. The gravitational constant and the Planck units. A simplification of the quantum realm. Physics Essays Vol 29, No 4, 2016.

[7] H. Cavendish. Experiments to determine the density of the Earth. Philosophical Transactions of the Royal Society of London, (part II), 88, 1798.

[8] M. E. McCulloch. Gravity from the uncertainty principle. Astrophysics and Space Science, 349(2), 2014.

[9] E. G. Haug. Can the Planck length be found independent of big G? Applied Physics Research, 9(6), 2017.

[10] E. G. Haug. Planck mass measured totally independent of big G utilizing McCulloch-Heisenberg Newtonian equivalent gravity. preprints.org, 2018.

[11] M. E. McCulloch. Galaxy rotations from quantised inertia and visible matter only. Astrophys Space Science,
$362,2017$.

[12] W. Heisenberg. Über den anschaulichen inhalt der quantentheoretischen kinematik und mechanik. Zeitschrift für Physik, (43):172-198, 1927.

[13] G. S. Bisnovatyi-Kogan. Checking the variability of the gravitational constant with binary pulsars. International Journal of Modern Physics D, 15(07), 2006.

[14] B. Fixler, G. T. Foster, J. M. McGuirk, and M. A. Kasevich. Atom interferometer measurement of the Newtonian constant of gravity. Science, 315, 2007.

[15] S. Galli, A. Melchiorri, G. F. Smoot, and O. Zahn. From Cavendish to Planck: Constraining Newton's gravitational constant with CMB temperature and polarization anisotropy. Physical Review D, 80, 2009.

[16] G. Rosi, F. Sorrentino, L. Cacciapuoti, M. Prevedelli, and G. M. Tino. Precision measurement of the Newtonian gravitational constant using cold atoms. Nature, 510, 2014 .

[17] F. Scholkmann. Indications for a diurnal and annual variation in the anisotropy of diffusion patterns: A reanalysis of data presented by J. Dai (2014, Nat. Sci.). Progress in Physics, 10, 2014.

[18] M. E. McCulloch. Quantised inertia from relativity and the uncertainty principle. EPL (Europhysics Letters), 115(6), 2016.

[19] M. Planck. Naturlische Masseinheiten. Der Königlich Preussischen Akademie Der Wissenschaften, p . 479, 1899.

[20] M. Planck. Vorlesungen Über die Theorie der Wärmestrahlung. Leipzig: J. A. Barth, p. 163, see also the English translation "The Theory of Radiation" (1959) Dover, 1906.

[21] B. P. Kibble, J. H. Sanders, and A. H. Wapstra. A measurement of the gyromagnetic ratio of the proton by the strong field method. Atomic Masses and Fundamental 
Constants, 5, 1975.

[22] M. Stock. The Watt balance: Determination of the Planck constant and redefinition of the kilogram. Philosophical Transactions of the Royal Society, 369:39363953, 2011.

[23] I. A. Robinson and S. Schlamminger. First determination of the Planck constant using the Watt balance. Forthcoming, Metrologia, 2016.

[24] E. H.. Kennard. Zur quantenmechanik einfacher bewegungstypen". Zeitschrift für Physik, (44):326-352, 1927.

[25] E. G. Haug. Revisiting the derivation of Heisenberg's uncertainty principle: The collapse of uncertainty at the Planck scale. https://www.preprints.org 201805.0258, 2018.

[26] S. Lan, P. Kuan, B. Estey, D. English, J. M. Brown, M. A. Hohensee, and Müller. A clock directly linking time to a particle's mass. Science, 339, 2013.

[27] D. Dolce and A. Perali. On the Compton clock and the undulatory nature of particle mass in graphene systems.
The European Physical Journal Plus, 130(41), 2015.

[28] E. G. Haug. The Pauli objection addressed in a logical way. Working paper Norwegian University of Life Sciences, 2017.

[29] W. Pauli. Die allgemeinen prinzipien der wellenmechanik. Springer, Berlin, p.84, 190.

[33] J. Leon and L. Maccone. The Pauli objection. The Foundations of Physics, 47(12), 2017.

[31] E. A. Galapon. Self-adjoint time operator is the rule for discrete semibounded Hamiltonians. Proceedings of the Royal Society of London A: Mathematical, Physical, and Engineering Sciences, 458(2027), 2002.

[32] E. A. Galapon. Pauli's theorem and quantum canonical pairs: the consistency of a bounded, self-adjoint time operator canonically conjugate to a Hamiltonian with nonempty point spectrum. Philosophical Transactions of the Royal Society of London, 458(2018), 2002.

[33] J. Leon and L. Maccone. The Pauli objection. The Foundations of Physics, 47(12), 2017. 